\title{
Study of Lithograph Characteristics of Black Matrix (BM) Resist
}

\author{
Atsushi Sekiguchi ${ }^{12^{*}}$, Tomoki Nishino ${ }^{1}$, Hiroko Minami², \\ Yoko Matsumoto ${ }^{2}$, and Yan $\mathrm{Kai}^{3}$ \\ ${ }^{1}$ Litho Tech Japan Corp., 2-6-6-201 Namiki, Kawaguchi, Saitama 332-0034, Japan \\ ${ }^{2}$ Ritsumeikan University, 1-1-1 Noji-higashi, Kusatsu, Shiga 525-8577, Japan \\ ${ }^{3}$ ShenZhen RongDa Photosensitive Science \& Technology Co., Ltd., \\ Floor 1-3, R\&D Building of Lixin Lake No.1 Science and Technology Industrial Park, \\ Fuyong Town, Baoan District, Shenzhen, China \\ *Sekiguchi-pdg@ltj.co.jp
}

\begin{abstract}
A color filter (CF) for displays consists of color resists, transmitting red (R), green (G), and blue (B) light, deposited onto a pattern of black matrix (BM) film formed on a thin glass substrate. The BM prevents optical leaks while producing the color black and prevents the mixing of color resists in adjacent pixels of the matrix. The filter also has a structure that forms the ITO (transparent electrode) film, which serves as the common electrode for the TFT array substrate. The BM resist must have high optical density (OD) to prevent optical leaks. Manufacturing goals typically include reducing the unit cost of panels and ensuring high-sensitivity panel design to allow the high throughput production of large panels. The emergence of $8 \mathrm{~K}$ and $16 \mathrm{~K}$ technologies in recent years has generated demand for ever higher resolutions, which, in turn, has driven demand for thinner films capable of achieving greater resolution and OD. Thus, the development of BM resists must now meet the highest technological standards. Responding to these issues involves identifying BM lithography characteristics. As a first step, we focused on the lithographic properties of BM resists, particularly on development characteristics. However, it is difficult for conventional development monitors to measure development rates of BM resists. Here we report on an analysis performed by a development monitor based on the QCM method.
\end{abstract}

Keywords: Black matrix, Color filter, TFT substrate, OD, QCM method, Development rate measurement, Lithographic simulation

\section{Introduction}

A color filter (CF) for displays consists of color resists that transmit red $(\mathrm{R})$, green $(\mathrm{G})$ and blue $(\mathrm{B})$ light. The resists are deposited onto a black matrix (BM) pattern formed on a thin glass substrate to prevent optical leaks while producing the color black and to prevent the mixing of color resists in adjacent pixels in the matrix (Fig. 1). The filter also incorporates a structure that forms an ITO (transparent electrode) film, which serves as the common electrode for the TFT array substrate [1]. The BM resist must have high OD value to prevent optical leaks [2]. Manufacturing goals include reducing the unit cost of panels and high-sensitivity panel design, thereby allowing the high throughput production of large panels [2]. Recent years have seen growing demand for ever higher resolution to satisfy the requirements of $8 \mathrm{~K}$ and $16 \mathrm{~K}$ technologies. This, in turn, has generated growing demand for thinner films capable of achieving higher resolution while exhibiting higher OD [3]. In general, the thickness of a BM film and its OD value are approximately $1 \mu \mathrm{m}$ and $4.6 \mu^{-1}$, respectively. Recent years have seen growing demand for higher sensitivity to improve display quality, leading in turn to higher backlight luminance and the problem of optical leaks in the CF. One solution has been to generate a more gradual taper profile by decreasing the thickness of the BM film. Emerging requirements at the mass 
production level currently call for a film thickness of $0.6 \mu \mathrm{m}$ and an OD value of $5 \mu \mathrm{m}^{-1}$ or higher. Although a high-OD design can suppress RGB film upsurge, the development of BM resists will require the highest levels of technological expertise.

To overcome these issues, we must first identify the characteristics of BM lithography. As a first step, we focused on the lithographic properties of the BM resists, particularly on development characteristics. Development rate measurements of resists are typically performed by the RDA method [4] based on optical interference. High OD values make it extremely difficult to obtain light interference for BM. Thus, we used the QCM method [5-7] for the development monitor in our evaluation and analysis of BM development rates. The following is a report on the results.

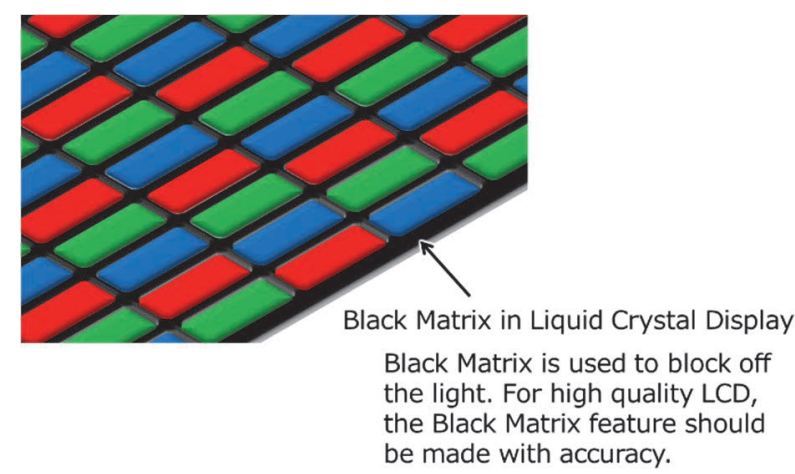

Fig. 1. The structure of a color filter and the role of BM.

\section{QCM method}

Analysis of resist development is commonly performed by the RDA method [4], which relies on optical interference. However, due to high OD values, it is extremely difficult to obtain optical interference for BM. This is because the light to be measured from the monitor is absorbed by the resist, eliminating reflected light. Thus, we applied the QCM method to evaluate and analyze BM development rates. The QCM method [5-7] allows us to measure the mass of the resist using a QCM (quartz crystal microbalance) substrate during development. If the resin density is known, we can convert the measured mass into thickness. Thus, this method allows analysis of both the dissolution behavior of resists and the swelling that occurs during development. Figure 2 shows an external view of RDA-Qz3 (manufactured by Litho Tech Japan), a resist development analyzer unit using QCM.

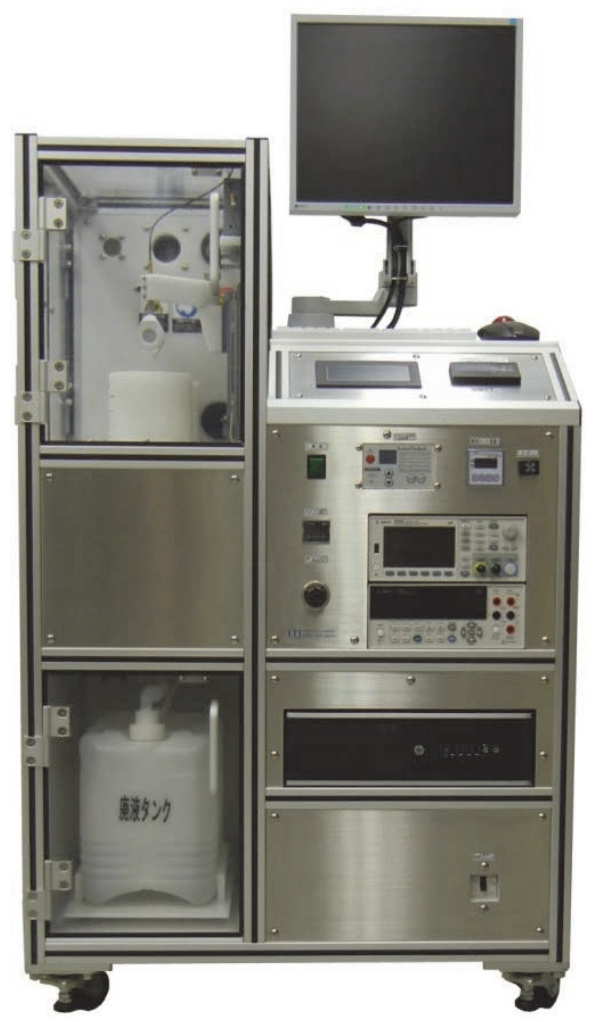

Fig. 2. External view of the RDA-Qz3.

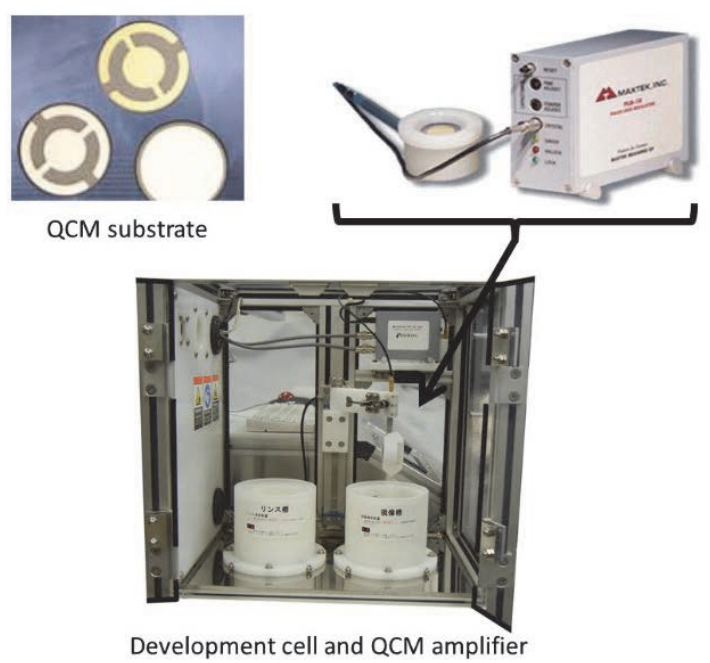

Fig. 3. QCM substrate and development cell.

Figure 3 shows an external view of the QCM substrate holder and the development cell.

Here, we will provide a brief summary of measurement principles based on QCM. A QCM substrate features an $\mathrm{Au}$ electrode attached to each 


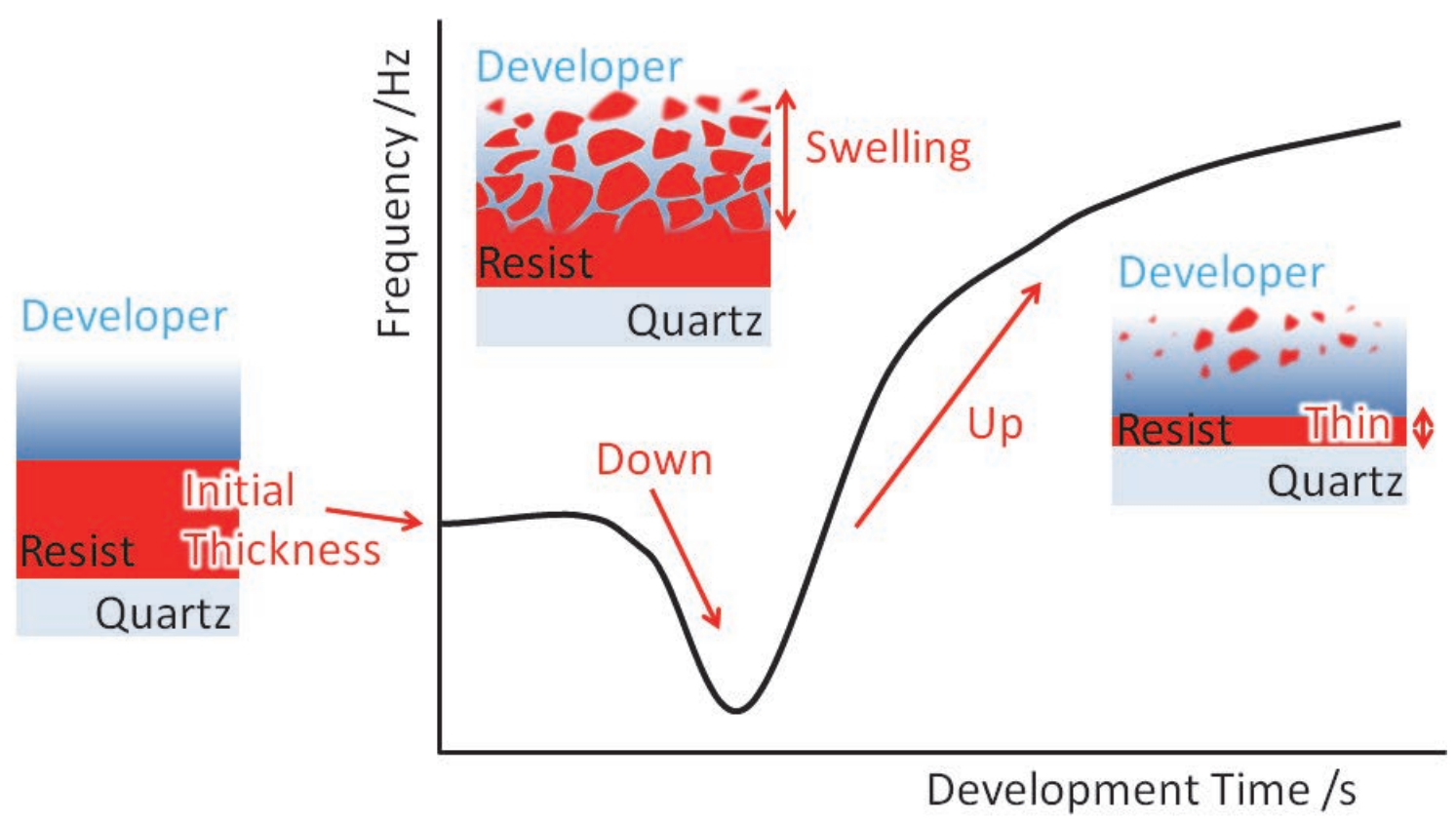

Fig. 4. Swelling and dissolution resist behavior and changes in the frequency of resonance.

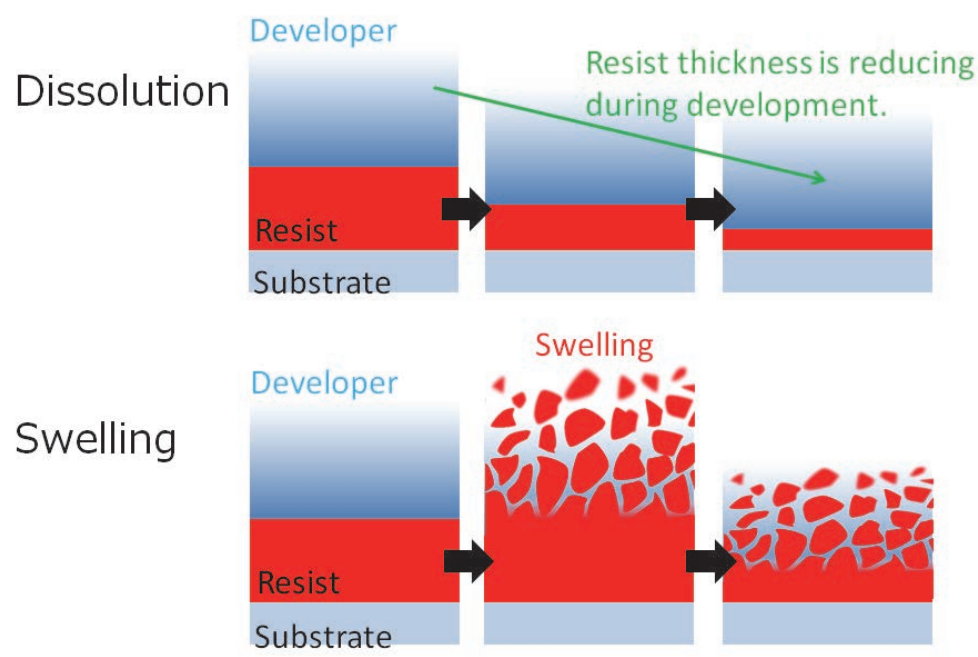

Fig. 5. Schematic models illustrating the simple dissolution of a resist film and dissolution accompanied by swelling during development.

of its surfaces. When a certain voltage is applied to the QCM substrate, it begins to resonate at the specific frequency of oscillation of the quartz crystal, called the frequency of resonance. When a resist is applied to the QCM substrate, the frequency of resonance decreases due to the increase in mass. If the resist-coated QCM substrate is immersed in developer solution, any swelling in the resist film will further become heavier due to the film absorbing the solution and lower the frequency of resonance. On the other hand, if the resist reverts to dissolution, the frequency of resonance increases; in the case of complete dissolution, the frequency of resonance will return to that of the substrate itself. Figure 4 shows this change in the frequency of resonance as development progresses.

Next, we will briefly describe swelling. Dissolution corresponds to the layer-by-layer exfoliation of the resist film. During dissolution, the frequency of resonance increases. In contrast, during swelling, the resist film absorbs the developer solution and expands in volume as it separates into clusters of molecules, which are 
eventually dispersed in the developer solution. When the resist film retains the developer solution, the frequency of resonance decreases.

Figure 5 gives schematic models of these phenomena.

Using Sauerbrey's equation [8], we can convert the frequency of resonance into mass, which in turn, based on density, can be used to obtain the relationship between development times and film thickness.

$$
\Delta f=-\frac{2 f_{0}^{2}}{\sqrt{\rho_{Q} \mu_{Q}}} m^{\prime}
$$

Here, $\Delta f$ is the change in frequency, $f_{0}$ the frequency of resonance, $\rho_{Q}$ the density of the quartz crystal $\left(2.649 \mathrm{~g} / \mathrm{cm}^{3}\right), \mu_{Q}$ the effective modulus of elasticity $\left(2.947 \times 10^{10} \mathrm{~N} / \mathrm{m}^{2}\right)$, and $\mathrm{m}$ ' the area density $\left(\mathrm{kg} / \mathrm{m}^{2}\right)$. A $10 \mathrm{~Hz}$ change in a $5 \mathrm{MHz}$ crystal is easily measured. The mass density in this case is $0.18 \mu \mathrm{g} / \mathrm{cm}^{2}$. In the case of a thin film (e.g., organic film) with a density of $1,200 \mathrm{~kg} / \mathrm{m}^{3}$, this corresponds to a thickness of approximately 15 angstroms.

\section{Experimental conditions and measurement results}

This section presents the experimental conditions.
The resist used in the study was the RD-NBM320 $\mathrm{BM}$ resist manufactured by Shen Zhen Ronda. The pre-bake was performed at $100{ }^{\circ} \mathrm{C}$ for 180 seconds. The film thickness was $630 \mathrm{~mm}$. The exposure wavelength was set to $365 \mathrm{~nm}$ (the "i line"). The developer solution was prepared at 3 different $\mathrm{KOH}$ concentrations: $0.030 \%, 0.045 \%$, and $0.090 \%$. Exposures were made using the UVES-2000 photoprocess analyzer (Litho Tech Japan). Development was analyzed with an RDA-Qz3 (Litho Tech Japan). Film thickness measurements were made using an Alpha-Step (KLA-Tencor/D$500)$.

\subsection{Evaluation of Eth values}

We determined the Eth (exposer dose threshold) for sensitivity at three different developer solution concentrations. Here we define Eth once again.

In the case of negative resists, the unexposed film dissolves completely in the developer solution. At gradually increased exposure doses, a residual film forms after development. Eventually, a point is reached at which the developer solution does not dissolve the film. The Eth value is an indicator of resist sensitivity and is defined in this paper as the point at which the exposure dose becomes high enough to leave a residual film after development.

Figure 7 gives the Eth values at different developer solution concentrations. Development was performed by the dip oscillation method for 90 seconds at $23^{\circ} \mathrm{C}$.

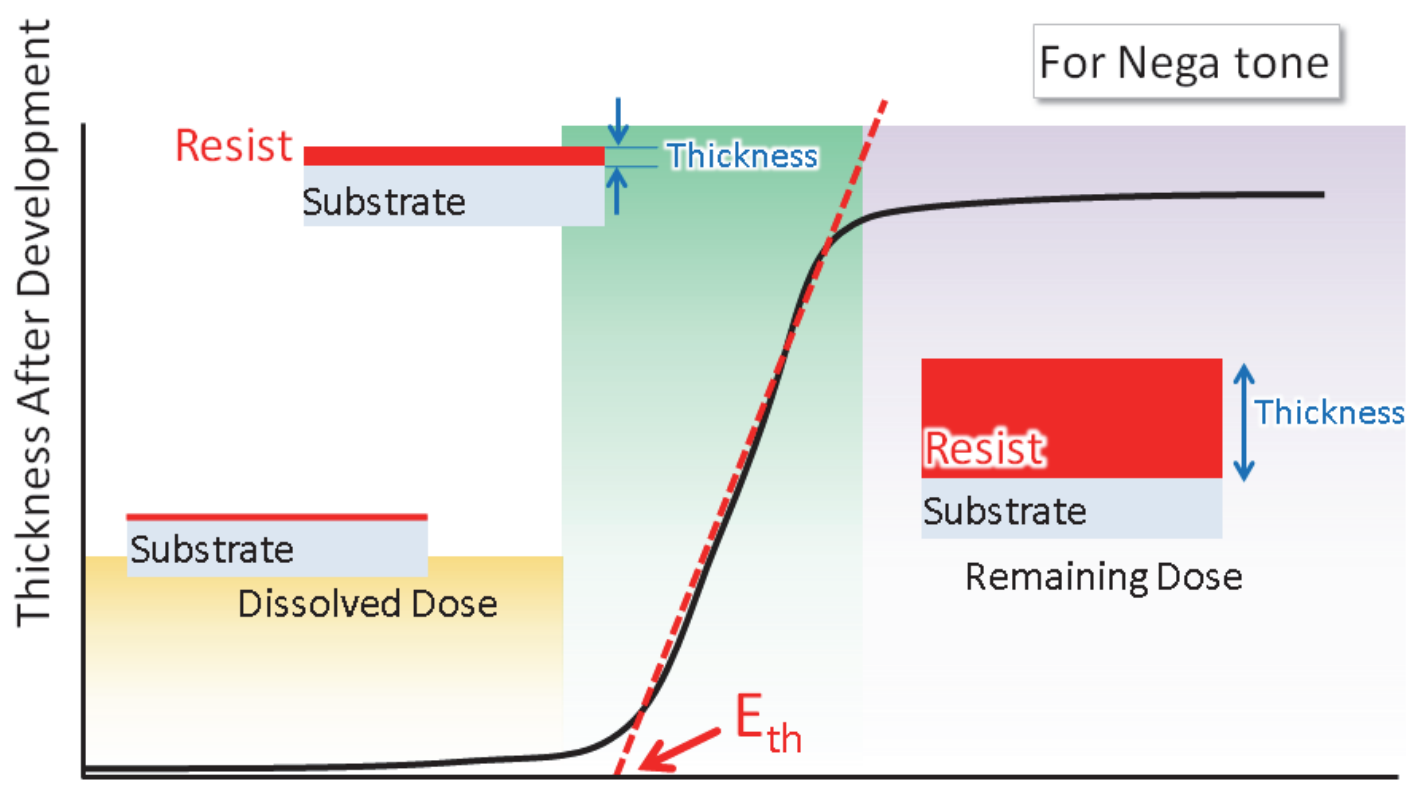

Exposure Dose

Fig. 6. Schematic diagram illustrating the Eth measurement principle during development. 


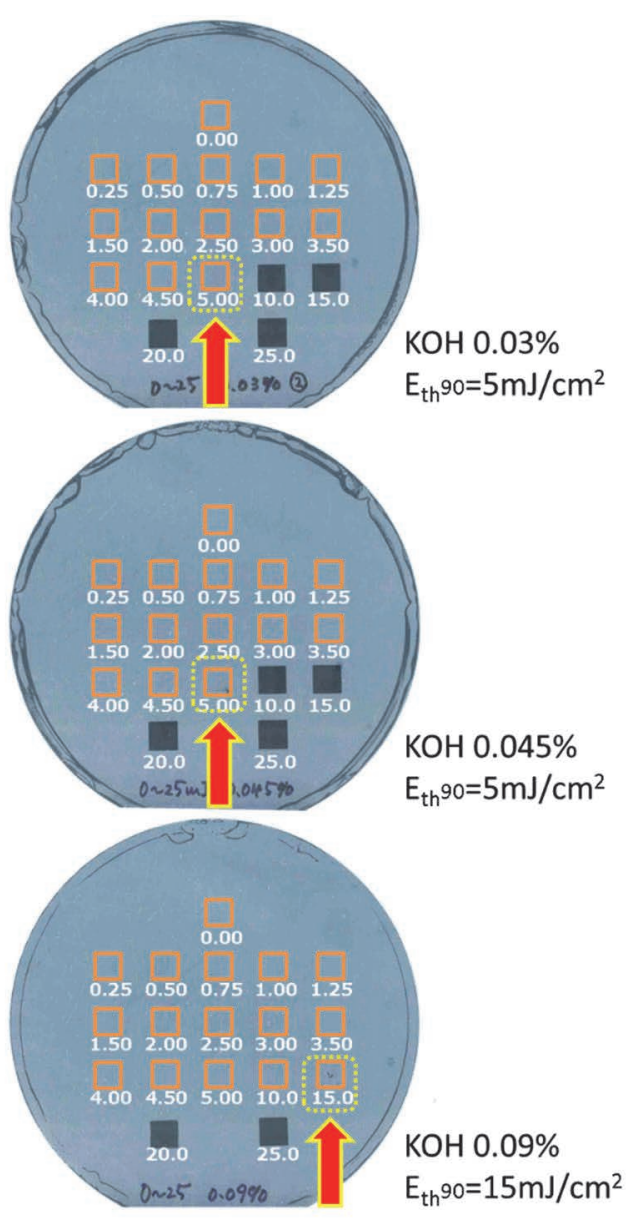

Fig. 7. Results of Eth measurement at different developer solution concentrations.

The results indicate Eth values are the same at 5 $\mathrm{mJ} / \mathrm{cm}^{2}$ at developer solution concentrations of $0.030 \%$ and $0.045 \%$, and $15 \mathrm{~mJ} / \mathrm{cm}^{2}$ at $0.090 \%$. Sensitivity is low. Figure 8 shows the film thickness after development for the developer concentration of $0.045 \%$. We see that swelling occurs at exposure doses of $6 \mathrm{~mJ} / \mathrm{cm}^{2}-50 \mathrm{~mJ} / \mathrm{cm}^{2}$.

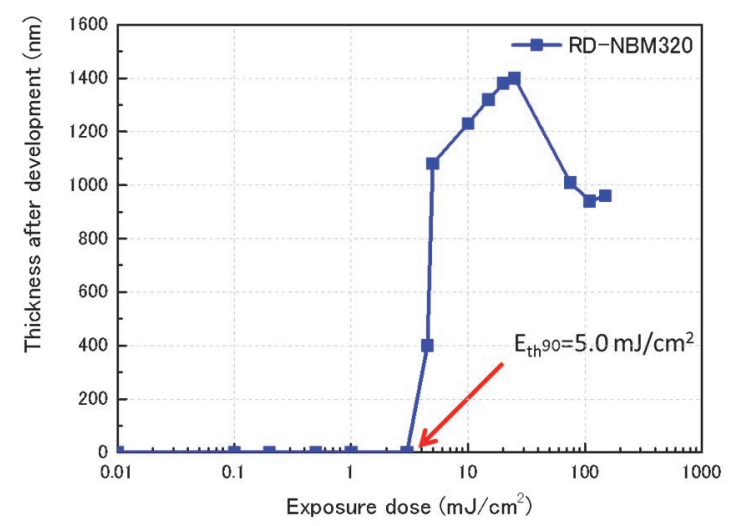

Fig. 8. Sensitivity curve and determination of Eth for $0.045 \%$ developer $\mathrm{KOH}$ concentration.
Figure 9 presents sensitivity curves. The horizontal axis gives the exposure dose in the sensitivity curve diagram; the vertical axis gives film thickness after development. The $\gamma$ value can be derived based on the slopes of the sensitivity curves.

The $\gamma$ value was found to be 6.26 at $\mathrm{KOH}$ concentrations of $0.030 \%$ and $0.045 \%$ and 6.13 at $0.090 \%$. We can conclude that the $\gamma$ value is approximately the same and around 6 , regardless of the $\mathrm{KOH}$ concentration of the developer solution.

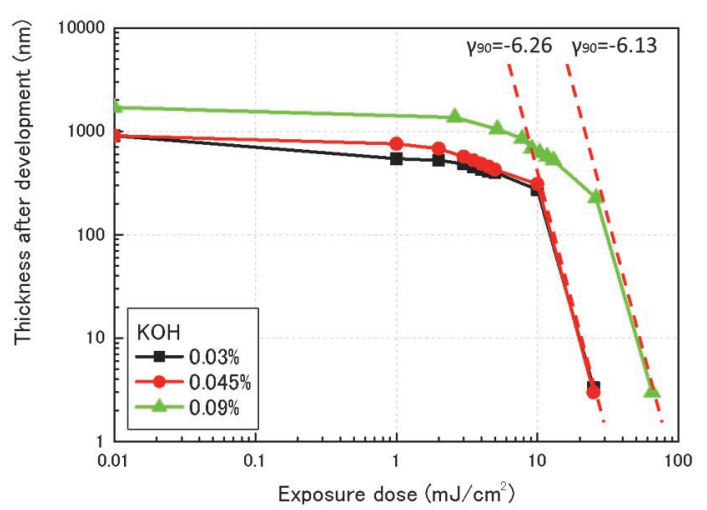

Fig. 9. Comparison of sensitivity curves (at development time of 90 seconds).

3.2. Evaluation of development characteristics by the QCM method

Figure 10 shows the frequency resonance measured as development time elapses for the developer $\mathrm{KOH}$ concentration of $0.045 \%$ by the QCM method. The exposure dose is $0 \mathrm{~mJ} / \mathrm{cm}^{2}-3.5$ $\mathrm{mJ} / \mathrm{cm}^{2}$.

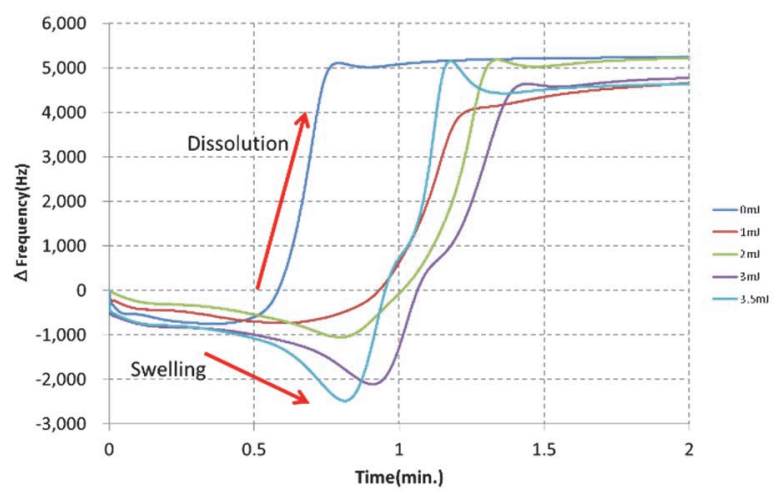

Fig. 10. Relationship between frequency of resonance and development time. $\mathrm{KOH}$ concentration: $0.045 \%$.

The frequency of resonance initially trends lower after development, but later reverts to an upward trend. This indicates swelling in the initial stages, switching later to dissolution. 
Figure 11 plots the relationship between resist film thickness and development time for different exposure doses. Swelling occurs at all developer $\mathrm{KOH}$ concentrations when development begins, eventually changing to dissolution. The maximum extent of the swelling was approximately $200 \%$. At the 90 second point after initiation of development for a $\mathrm{KOH}$ concentration of $0.045 \%$, the resist dissolves at $10 \mathrm{~mJ} / \mathrm{cm}^{2}$ but not at 20 $\mathrm{mJ} / \mathrm{cm}^{2}$.

(a)

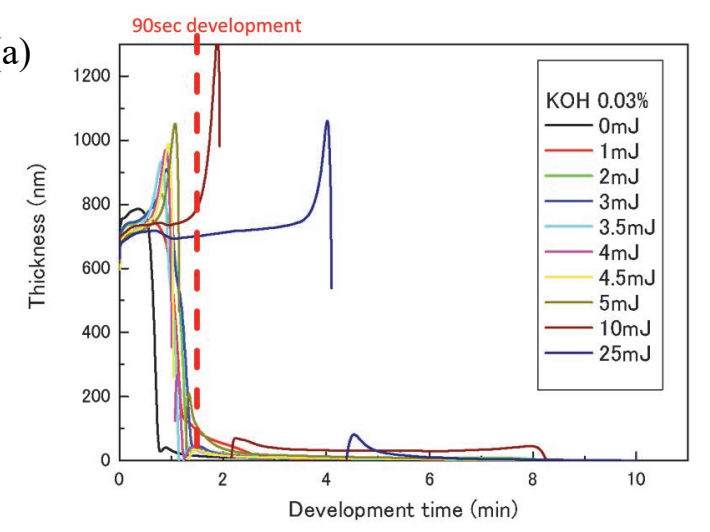

(b)

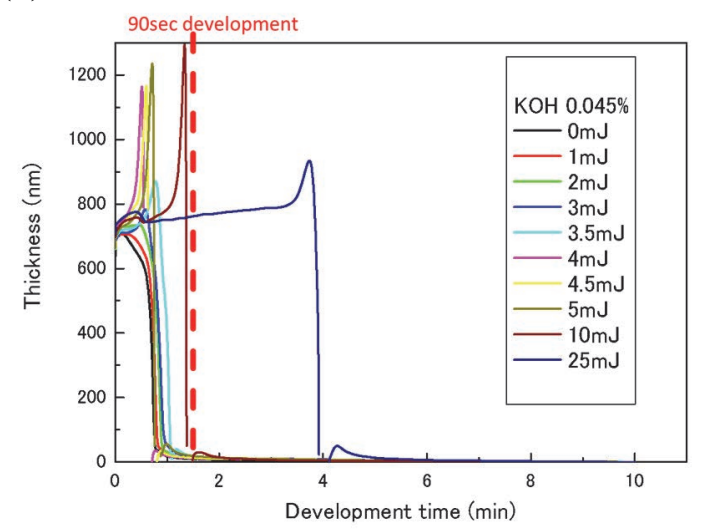

(c)

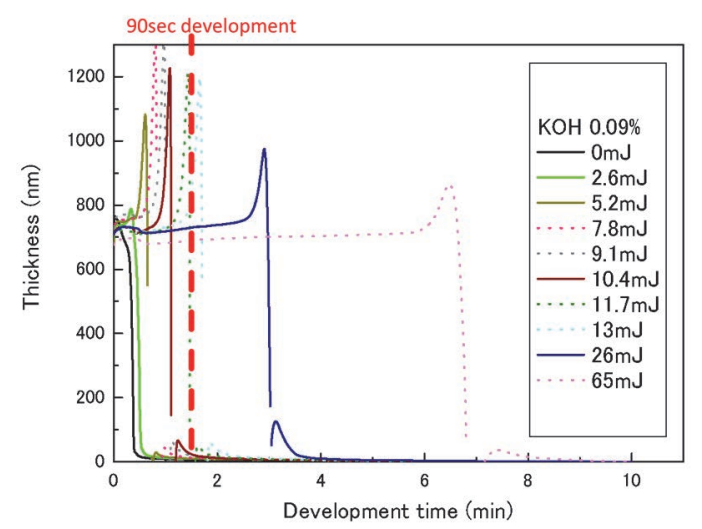

Fig. 11. Relationship between resist film thickness and development time for different exposure doses. $\mathrm{KOH}$ concentration: (a) $0.030 \%$, (b) $0.045 \%$, (c) $0.090 \%$.
Figure 12 shows the curves for the film thickness after development for difference developer solution concentrations at an exposure dose of $25 \mathrm{~mJ} / \mathrm{cm}^{2}$.

The resist begins to dissolves earlier at higher concentrations. That is, an increase in concentration accelerates resist dissolution.

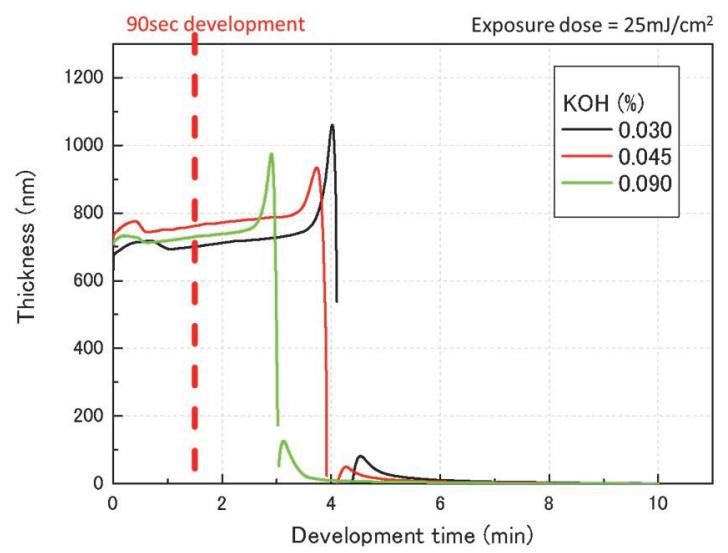

Fig. 12. Comparison of curves of film thickness after development at an exposure dose of $25 \mathrm{~mJ} / \mathrm{cm}^{2}$.

\subsection{Investigation by simulation}

Table 1 gives the Dill development parameters obtained from the QCM development data.

Figure 13 shows the results of simulation using the lithography simulation tool Prolith [9] after entering the development parameters from Table 1.

Table 1. Development parameters.

\begin{tabular}{c|c|ccc}
$\mathrm{KOH}(\%)$ & $\begin{array}{c}\mathrm{R}_{\max } \\
(\mathrm{nm} / \mathrm{s})\end{array}$ & $\begin{array}{c}\mathrm{R}_{\min } \\
(\mathrm{nm} / \mathrm{s})\end{array}$ & $M_{\text {th }}$ & $n$ \\
\hline 0.030 & 906.7 & 3.3 & 0.489 & 1.96 \\
0.045 & 910.5 & 3.0 & 0.521 & 2.02 \\
0.090 & 1720 & 3.0 & 0.511 & 1.88
\end{tabular}

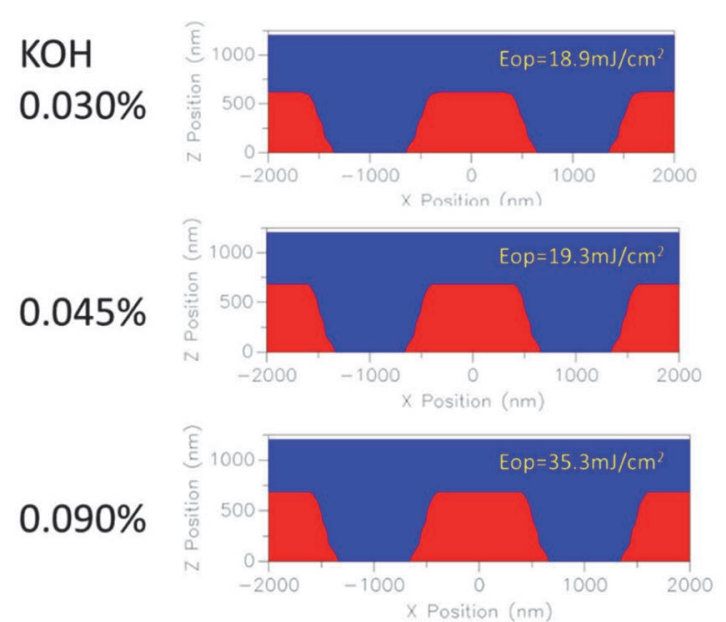

Fig. 13. Results of lithographic simulations for varied developer solution concentrations. 


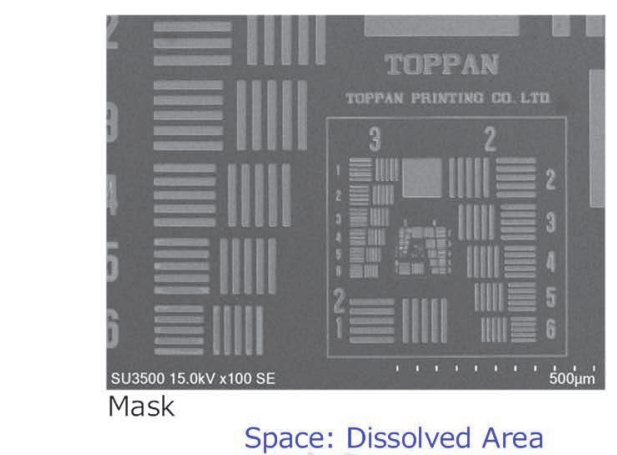

Exposure tool: LECET-500(LTJ)

Contact vacuum exposure

Wavelength: $365 \mathrm{~nm}$

Mask: Toppan Test Chart
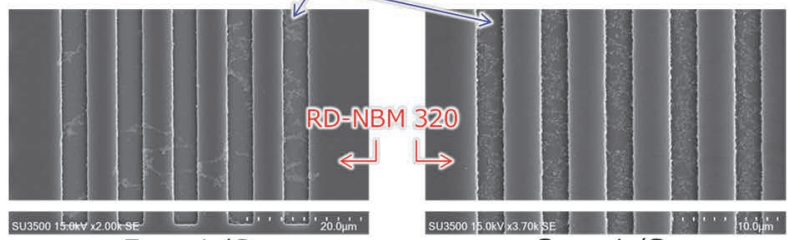

$3 \mu \mathrm{m} \mathrm{L} / \mathrm{S}$

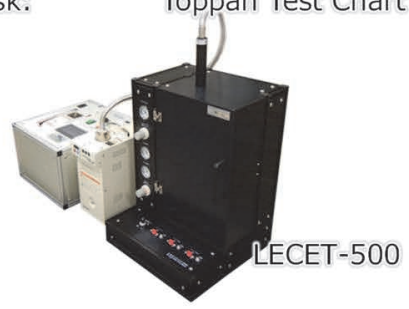

$5 \mu \mathrm{m}$ L/S

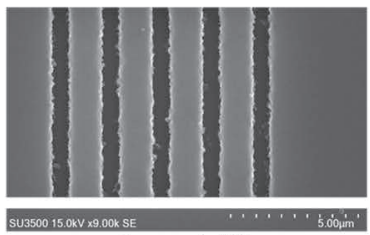

$1 \mu \mathrm{m} \mathrm{L} / \mathrm{S}$

Fig. 14. Results of masking and patterning.

The simulation conditions were exposure wavelength of $365 \mathrm{~nm}$, NA of 0.5 , and $\sigma$ of 0.5 . The pattern size consisted of $1 \mu \mathrm{m}$ lines. The development time was 90 seconds.

The results show no significant difference between the shapes of patterns with differences in developer solution concentration. Furthermore, at a concentration of $0.09 \%$, the $E$ op $=35.3 \mathrm{~mJ} / \mathrm{cm}^{2}$. This sensitivity is lower than that obtained at $\mathrm{KOH}$ concentrations of $0.030 \%$ and $0.045 \%$.

\section{Patterning investigation}

The above results suggest $\mathrm{KOH}$ concentrations of $0.030 \%-0.045 \%$ offer suitable resolution and sensitivity. We performed a patterning test using a $\mathrm{KOH}$ concentration of $0.045 \%$ as the standard condition. The other development parameters were $23{ }^{\circ} \mathrm{C}$ and 90 seconds.

The exposure unit was an LECT-500, a contact vacuum exposure unit for experiments manufactured by Litho Tech Japan [10-12]. A Toppan Test Chart was used as the mask for the evaluation. Figure 14 shows the results of

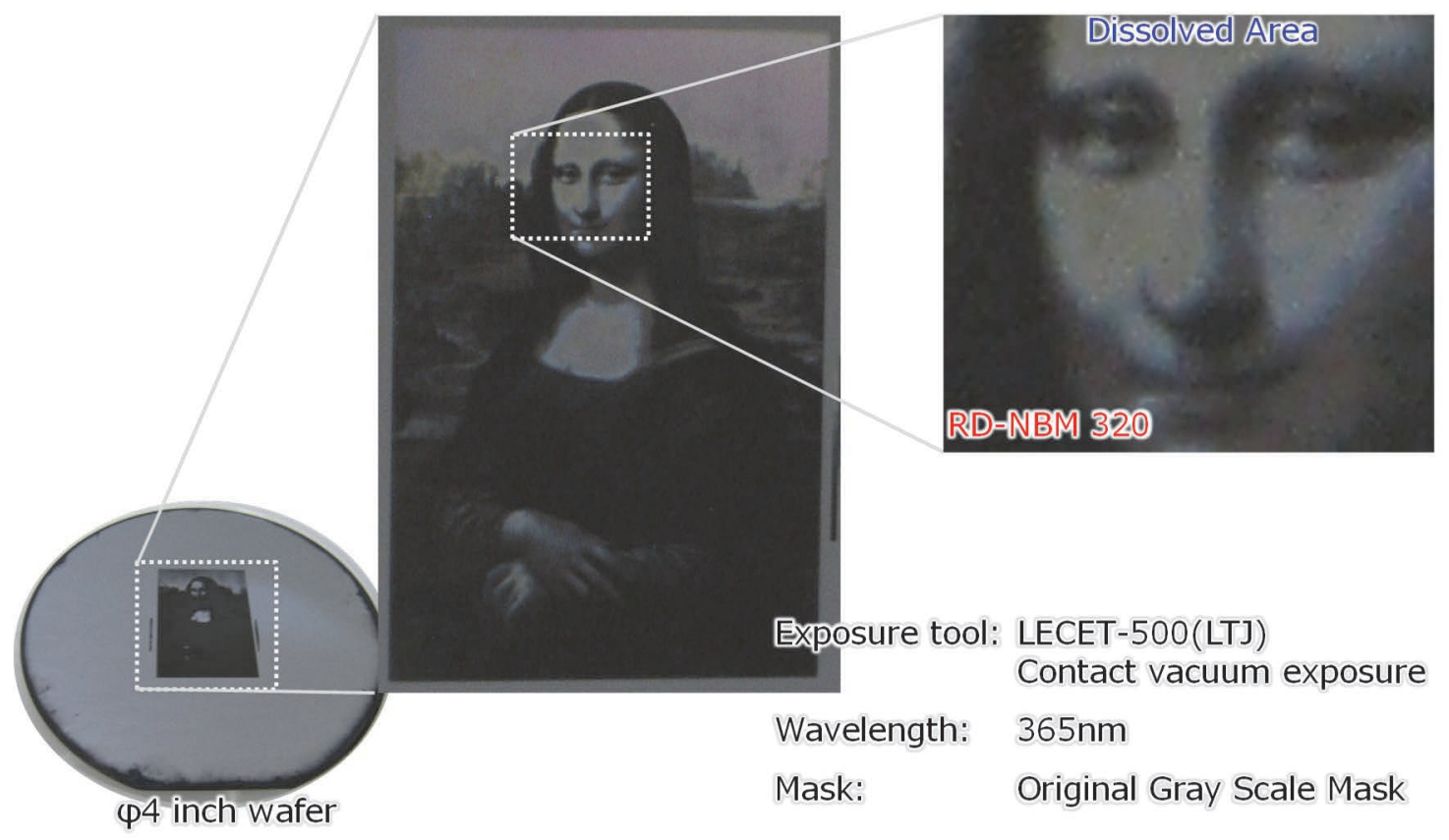

Fig. 15. Result of transcription using Mona Lisa gray mask. 
masking and patterning.

The developed patterns were observed using SEM. The results show that a $1 \mu \mathrm{m}$ line is resolved.

Finally, we performed a patterning test using a gray-scale mask (Mona Lisa pattern). Figure 15 gives the results.

A clear Mona Lisa pattern was obtained.

\section{Summary}

We examined the development characteristics of RD-MBN320, a BM resist for LCDs, by the QCM method. The results indicate the development of this resist accompanies swelling in the initial stages. We carried out simulations using development parameters calculated from development rate data obtained with the QCD method. The optimal $\mathrm{KOH}$ concentration in terms of sensitivity and resolution was determined to be $0.045 \%$. We then performed, patterning using a developer solution with a $\mathrm{KOH}$ concentration of $0.045 \%$. We found that the $1 \mu \mathrm{m}$ pattern was resolved. Future studies will target developer solution additives.

\section{Acknowledgements}

We are grateful to Hirohito Watanabe at Assist Navi for performing measurements of film thickness following development and to Tak-nam
Park at New Star International, who made the arrangements for the shipment of resist materials.

\section{References}

1. M. Arihara, J. Print. Sci. Technol., 49 (2012) 101 (in Japanese).

2. M. Shida and T. Kato, Monthly Display, 10 (2006) 31 .

3. M. Shida, LCD Components/Materials Outlook, (2009) 96 (in Japanese).

4. A. Sekiguchi, Y. Minami, and T. Matsuzawa, Electron. Commun. Jpn., Part II, 79 (1996) 51.

5. A. Sekiguchi, M. Isono, Y. Kono, and Y. Sensu, $J$. Photopolym. Sci. Technol., 17 (2004) 107.

6. S.-W. Lee, W. D. Hinsberg, and K. K. Kanazawa, Anal. Chem., 74 (2002) 125.

7. K. K. Kanazawa, Analyst, 130 (2005) 1459.

8. A. Sekiguchi, J. Photopolym. Sci. Technol., 23 (2010) 421.

9. A. Sekiguchi, J. Photopolym. Sci. Technol., 26 (2013) 479.

10. A. Sekiguchi, H. Konishi, and M. Isono, Proc. SPIE, 8325 (2012) 61.

11. C. A. Mack, T. Matsuzawa, A. Sekiguchi, and Y. Minami, Proc. SPIE, 2725 (1996) 34.

12. A. Sekiguchi, Y. Sensu, and Y. Miyake, Jpn. J. Appl. Phys., 42 (2003) 16. 